\title{
The development and implementation of a strategic framework to improve Aboriginal child development and wellbeing in far west NSW: a collaborative approach
}

\author{
Garth Alperstein $^{\mathrm{A}, \mathrm{B}}$ and Cathy S. Dyer ${ }^{\mathrm{A}}$ \\ ${ }^{\mathrm{A}}$ Maari Ma Health Aboriginal Corporation, Broken Hill \\ ${ }^{\mathrm{B} C o r r e s p o n d i n g ~ a u t h o r . E m a i l: ~ g a l p e r s t e i n @ g m a i l . c o m ~}$
}

\begin{abstract}
In 2005, the Maari Ma Chronic Disease Strategy of the Maari Ma Health Aboriginal Corporation in far west NSW identified the importance of addressing the fetal, infant and child origins of chronic disease in adults. In 2008 a process for developing a strategic framework to improve the development and wellbeing of children was initiated. The process incorporated all organisations involved with children. A multisectoral working group was established to facilitate the development of the strategic framework which was published in 2009 and a Project Officer was employed by Maari Ma to implement it. This included working with agencies that have the potential to affect the social determinants of health. It is anticipated that, in the medium to long term, this approach will contribute to reducing the rate of chronic disease in adulthood, and reduce the gap between Aboriginal and non-Aboriginal health outcomes.
\end{abstract}

in 2005. Part of the Strategy recognised the fetal and childhood origins of chronic disease in adults. At that time, the service did not have a similar comprehensive framework for child health. As a result, Maari Ma supported and facilitated the development of the Strategic framework to improve child development and wellbeing for Aboriginal children in the far west, which was published in March 2009.

This paper describes both the Maari Ma Chronic Disease Strategy and the development and implementation to date of the strategic Framework for children.

\section{The Maari Ma Chronic Disease Strategy}

The Strategy was based on a number of existing documents including Preventable Chronic Diseases Strategy (1999) from the Northern Territory Department of Health and Community Services, Chronic Disease Strategy, Enhanced Model of Primary Health Care (Queensland Health), National Chronic Disease Prevention Framework (2001) (National Public Health Partnership) and NSW Health's then draft NSW Aboriginal Chronic Disease Service Framework (2003).

The Maari Ma Chronic Disease Strategy was comprehensive and included evidence-based, best-practice early detection, prevention and management of chronic disease. The Strategy drew on current best practice in Australia and specifically noted that 'the origins of many chronic diseases are set in utero and early childhood (most notably through low birthweight, growth retardation and repeated childhood infections) and are worsened by lifestyle changes (weight gain, lack of physical activity and substance abuse).' It noted that 'the diseases and their risk factors are also inextricably linked with the broader socioeconomic determinants of health and quality of life, particularly education and employment. Lifestyle choices are often more reflective of unrelenting socio-environmental constraints rather than personal preferences. Therefore an integrated, intersectoral and whole-of-life approach is needed.' 


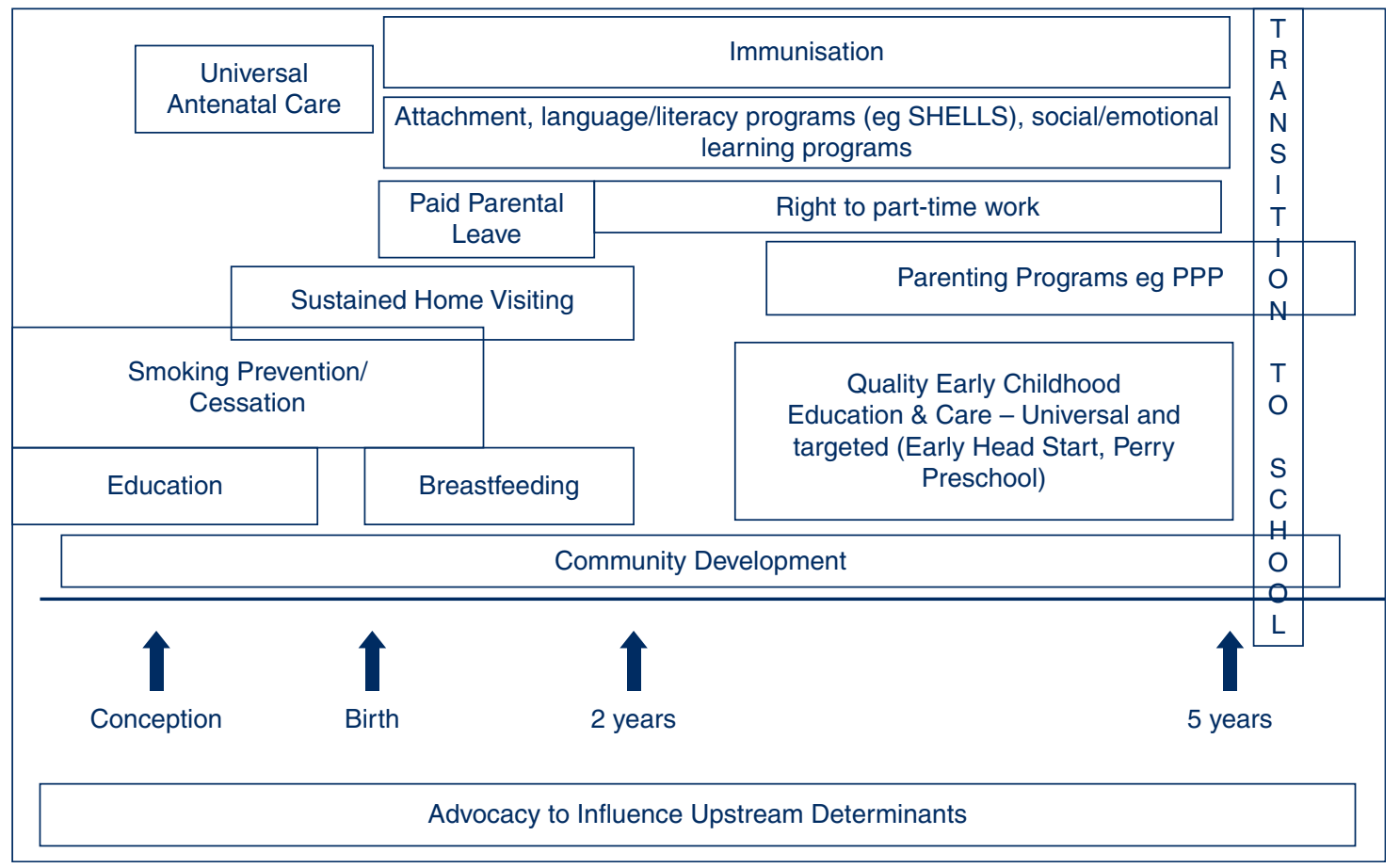

Figure 1. Key components of the strategic framework to improve child development and wellbeing for Aboriginal children in the far west of NSW, 2009.

Adapted from: Nossar V, Alperstein G. Key Intitiatives to achieve health gain for children. NSW Public Health Bull 1998; 9(11): 126-7.

Finally, the document recognised that while some improved outcomes would be seen in the short to medium term, as well as a reduction in long-term financial costs, the full impact of all the interventions would not be felt for some years. This was therefore a staged long-term strategy to overcome problems that have arisen over many decades.

\section{Strategic framework document}

With the recognition of the fetal, infant and early childhood determinants of chronic disease, Maari Ma undertook to develop a preventive and population-based approach to improving fetal, infant and early childhood development and wellbeing. Child development and wellbeing refer to physical, emotional, social and cognitive wellbeing. This concept is similar to the Aboriginal definition of health ${ }^{1}$ :

Health does not just mean the physical wellbeing of the individual but refers to the social, emotional, spiritual and cultural wellbeing of the whole community. This is a whole-of-life view and includes the cyclical concept of life-death-life.

There is well documented evidence that investment in promoting child development and wellbeing in the early years is more cost effective than addressing ill-health, poor social outcomes and educational deficits later in life. For example, for every $\$ 1$ spent per child on the Perry Preschool program, at 40-year follow-up, $\$ 17$ is returned to society $^{2}$; for every $\$ 1$ spent on the nurse home visiting program, at 15 -year follow-up, $\$ 5$ is returned to society. ${ }^{3}$

Using the same principles that informed the Families NSW strategy of multisectoral participation, a preventive, early intervention and outcomes focus, a population approach and evidence-based strategies, the process was initiated by organising a forum in Broken Hill in May 2008 which was attended by all organisations and agencies involved with children. Presentations of the Framework strategy, including the principles, rationale and supporting evidence, were given separately to all involved communities in the region. A multisectoral working group comprising health (Aboriginal community controlled and mainstream), education, preschool, community services, police and Non Government Organisations (NGOs) has been meeting regularly to progress implementation of priority strategies.

From previously published research, the key strategies to improve child development and wellbeing from a prevention, early intervention and promotion of health perspective are summarised in Figure 1.

Using those strategies as a guide, a framework was developed for the local Aboriginal population and the services engaging with them. Based on the research, the strategies in the Framework have the potential to lead to improved child development and wellbeing outcomes in the short to medium term, a reduction in some chronic disease 
Box 1. Maari Ma documents supporting the strategic framework to improve Aboriginal child development and wellbeing

- Maari Ma Chronic Disease Strategy (2005)

- Strategic framework to improve child development and wellbeing for Aboriginal children in the far west (2009)

- Health, development and wellbeing in far western NSW. A picture of our children (2009)

These documents are available at: http://maarima.com.au under Publications.

in adulthood, as well as a reduction in long-term financial costs. However, like the chronic disease strategy, the full impact of all the interventions will not be felt for some years.

\section{Monitoring outcomes}

In order to monitor the impact of the strategies, a baseline set of data was developed and Health, development and wellbeing in far western NSW. A picture of our children was published in September 2009 (Box 1). This report was modelled on the Australian Institute of Health and Welfare's national indicators of children's health and wellbeing $^{4}$ and included health, educational and social indicators to reflect the broad approach being taken to child development and wellbeing. Where possible, data from far western NSW were used to compare outcomes for local Aboriginal children and their families with Aboriginal populations elsewhere in NSW, and NSW populations in general. In cases where local data could not be obtained, in regional (the former Greater Western Area Health Service) data were used. These key indicators will be collected every 5 years to monitor progress and the impact of strategies that are implemented.

A smaller subset of 14 key indicators was chosen to monitor the gap in outcomes. The desired outcome is for the gap to be equal to or less than 1, indicating that the result for the Aboriginal population in far western NSW is the same as, or better than, the whole NSW population.

\section{Implementation of the Framework}

The implementation of the strategies in the Framework is a long-term initiative. The same multisectoral working group has been reconvened to facilitate and drive the implementation. Since the completion of the Framework, the following changes and new initiatives have occurred:

- The Maari Ma 'Healthy Start' program, a preventive schedule of clinic-based and home visits by midwives, child and family nurses and primary health care and Aboriginal health workers from the antenatal period to school entry, was revised to reflect the most up-todate research evidence related to prevention, early intervention, and screening and surveillance. Most recently, general practitioners employed by Maari Ma have been incorporated into the schedule.

- A perinatal, infant, child and adolescent psychiatrist was engaged to build the capacity of antenatal and child and family staff in mental health in pregnancy, bonding and attachment between mothers and babies, promotion of resilience, and management of common childhood behavioural problems. This capacity building has entailed quarterly training sessions and clinical supervision of staff.

- In order to promote a collaborative approach and work with agencies and organisations influencing the social determinants of health of families and young children, a full time Early Years Project Leader was employed in February 2010. The Project Leader has focused on three key areas:

a. Establishing networks: Local Child and Family networks have been established in Broken Hill, Menindee and Wilcannia to foster collaborative working relations between all agencies working with families with young children.

b. Early childhood education and care: Training in literacy, infant mental health, science and maths has been coordinated for health staff, NGOs and early childhood educators; an introduction to children's services training for the Wilcannia Intensive Supported Playgroup staff has been delivered; an ongoing Early Years Discussion Group comprising early childhood educators working in child care and preschools to share knowledge and ideas around the National Quality Framework for Early Childhood Education and Care has been facilitated; early literacy through community events has been promoted; a 'Book-in-Homes' program has distributed books in Wilcannia and Menindee to families with infants and young children $0-3$ years of age.

c. Parenting: The development of Kiilalaana martamarri (Growing up really big Barkindjii) in conjunction with NSW Family and Community Services (Aboriginal Child, Youth and Family Strategy), a resource on child development for parents is being facilitated; the Broken Hill Healthy Start playgroup for Aboriginal families with young preschool-aged children which operates on a weekly basis is being initiated and led.

After 3 years of implementation, a formal evaluation of the implementation process will be undertaken to identify achievements and gaps.

\section{Discussion}

Health services alone cannot optimise the health, development and wellbeing of Aboriginal children; it is also necessary to influence the socio-economic determinants. In addition to taking a population approach, the strategic framework is an attempt to influence the socio-economic 
determinants by working collaboratively with other government and non-government agencies that have the potential to affect some of the socio-economic determinants of health, child development and wellbeing.

Many of the strategies in Figure 1 are currently being implemented, fully or partially, such as immunisation, home visiting programs, community development and early literacy. Others require further funding, planning and implementation. Advocacy to influence the socioeconomic determinants and higher level policies such as paid parental leave is an ongoing process and the responsibility of all those attempting to improve the health and wellbeing of populations, particularly the most disadvantaged.

The implementation of the Framework is a long-term process and dependant on many factors including available funding, staffing levels and commitment by those involved.

\section{Conclusion}

It is anticipated that progressive improvements in health, educational and social outcomes will be achieved, and will eventually result in bridging the health gap between
Aboriginal and non-Aboriginal outcomes in the far west of NSW by:

- taking a collaborative approach

- involving all organisations that engage with Aboriginal families and potentially have an effect on child health and wellbeing

- focusing on prevention and early intervention.

\section{References}

1. National Health and Medical Research Council. Promoting the health of Indigenous Australians. A review of infrastructure support for Aboriginal and Torres Strait Islander health advancement. Final report and recommendations. Canberra: NHMRC; 1996, part 2:4.

2. Olds DL, Eckenrode J, Henderson CR, Kitzman H, Powers J, Cole $\mathrm{R}$ et al. Long term effects of home visitation on maternal life course and child abuse and neglect: fifteen year follow up of a randomised trial. JAMA 1997; 278: 637-43. doi:10.1001/ jama.1997.03550080047038

3. Schweinhart LJ, Montie Xiang JZ, Barnett WS, Belfield CR, Nores M. Lifetime effects: the High/Scope Perry Preschool Study through age 40. Ypsilanti, Mich: High/Scope Press; 2005.

4. Australian Institute of Health and Welfare. Key national indicators of children's health, development and wellbeing indicator framework for 'A picture of Australia's children 2009'. Cat. no. AUS 100. Canberra: AIHW; 2008. 